\title{
A note on the rational cuspidal curves
}

\author{
Piotr Nayar, Barbara Pilat
}

\begin{abstract}
In this short note we give an elementary combinatorial argument, showing that the Conjecture of J. Fernández de Bobadilla, I. Luengo, A. Melle-Hernández, A. Némethi (see [BL, Conjecture 1.2) follows from Theorem 5.4 in $\mathrm{BL}$ in the case of rational cuspidal curves with two critical points.
\end{abstract}

2010 Mathematics Subject Classification. Primary 14H50; Secondary 14B05, 57M25, 57R58.

Key words and phrases. Rational cuspidal curve, Alexander polynomial, infimum convolution.

\section{Introduction}

In this short note we deal with irreducible algebraic curves $C \subset \mathbb{C} P^{2}$. Such a curve has a finite set of singular points $\left\{z_{i}\right\}_{i=1}^{n}$ such that a neighbourhood of each singular point intersects $C$ in a cone on a link $K_{i} \subset S^{3}$. We would like to know what possible configurations of links $\left\{K_{i}\right\}_{i=1}^{n}$ arise in this way. We consider only the case in which each $K_{i}$ is connected (in this case $K_{i}$ is a knot), and thus $C$ is a rational curve, meaning that there is a rational surjective map $\mathbb{C} P^{1} \rightarrow C$. Such a curve is called rational cuspidal. We refer to [M] for a survey on rational cuspidal curves.

Suppose that $z$ is a cuspidal singular point of a curve $C$ and $B$ is a sufficiently small ball around $z$. Let $\Psi(t)=(x(t), y(t))$ be a local parametrization of $C \cap B$ near $z$. For any polynomial $G(x, y)$ we look at the order at 0 of an analytic map $t \mapsto G(x(t), y(t)) \in C$. Let $S$ be the set of integers, which can be realized as the order for some $G$. Then $S$ is a semigroup of $\mathbb{Z}_{\geq 0}$. We call it the semigroup of the singular point, see [W] for the details and proofs. The gap sequence, $G=\mathbb{Z}_{\geq 0} \backslash S$, has precisely $\mu / 2$ elements, where the largest one is $\mu-1$. Here $\mu$ stands for the Milnor number. Assume that $K$ is the 
link of the singular point $z$. The Alexander polynomial of $K$ is of the form

$$
\Delta_{K}(t)=\sum_{i=0}^{2 m}(-1)^{i} t^{n_{i}}
$$

where $\left(n_{i}\right)_{i=0}^{2 m}$ form an increasing sequence with $n_{0}=0$ and $n_{2 m}=2 g$, for $g$ being the genus of $K$. Writing $t^{2 n_{i}}-t^{2 n_{i-1}}=(t-1)\left(t^{2 n_{i}-1}+t^{2 n_{i}-2}+\ldots+\right.$ $t^{2 n_{i-1}}$ ) yields the representation

$$
\Delta_{K}(t)=1+(t-1) \sum_{j=1}^{k} t^{g_{j}}
$$

for some finite sequence $0<g_{1}<g_{2}<\ldots<g_{k}$. We have the following lemma (see [W], Exercise 5.7.7), which relates the Alexander polynomial to the gap sequence of a singular point.

Lemma 1. The sequence $g_{1}, \ldots, g_{k}$ in (1) is the gap sequence of the semigroup of the singular point. In particular, $k=|G|=\mu / 2$, where $\mu$ is the Milnor number, so $|G|$ is the genus.

If we write $t^{g_{j}}=(t-1)\left(t^{g_{j}-1}+t^{g_{j}-2}+\ldots+1\right)+1$, we obtain

$$
\Delta_{K}(t)=1+(t-1) g(K)+(t-1)^{2} \sum_{j=0}^{\mu-2} k_{j} t^{j},
$$

where $k_{j}=|\{m>j: m \notin S\}|$. This motivates the following definition.

Definition. For any finite increasing sequence of positive integers $G$ we define

$$
I_{G}(m)=\left|\left\{k \in G \cup \mathbb{Z}_{<0}: k \geq m\right\}\right|,
$$

where $\mathbb{Z}_{<0}$ is the set of negative integers. We shall call $I_{G}$ the gap function, because in most applications $G$ will be a gap sequence of some semigroup.

Clearly, for $j=0,1, \ldots, \mu-2$ we have $I_{G}(j+1)=k_{j}$.

In [FLMN] the following conjecture was proposed.

Conjecture 1. Suppose that the rational cuspidal curve $C$ of degree d has critical points $z_{1}, \ldots, z_{n}$. Let $K_{1}, \ldots, K_{n}$ be the corresponding links of singular points and let $\Delta_{1}, \ldots, \Delta_{n}$ be their Alexander polynomials. Let $g$ be the genus of $K$. Let $\Delta=\Delta_{1}, \ldots, \Delta_{n}$, expanded as

$$
\Delta(t)=1+\frac{(d-1)(d-2)}{2}(t-1)+(t-1)^{2} \sum_{j=0}^{2 g-2} k_{l}
$$


Then for any $j=0, \ldots, d-3$ we have $k_{d(d-j-3)} \leq(j+1)(j+2) / 2$, with equality for $n=1$.

This conjecture was verified in the case $n=1$ by Borodzik and Livingston, see [BL].

We define the infimum convolution of two functions.

Definition. Let $I_{1}, I_{2}, \ldots, I_{n}: \mathbb{Z} \rightarrow \mathbb{Z}_{\geq 0}$. We define

$$
\left(I_{1} \diamond I_{2} \ldots \diamond I_{n}\right)(k)=\min _{\substack{k_{1}, k_{2}, \ldots, k_{n} \in \mathbb{Z} \\ k_{1}+k_{2}+\ldots+k_{n}=k}}\left(I_{1}\left(k_{1}\right)+I_{2}\left(k_{2}\right)+\ldots+I_{n}\left(k_{n}\right)\right) .
$$

In BL] the authors gave the proof of the following theorem.

Theorem 1. ([BL], Theorem 5.4) Let $C$ be a rational cuspidal curve of degree $d$. Let $I_{1}, \ldots, I_{n}$ be the gap functions associated to each singular point on $C$. Then for any $j \in\{-1,0, \ldots, d-2\}$ we have

$$
I_{1} \diamond I_{2} \diamond \ldots \diamond I_{n}(j d+1)=\frac{1}{2}(j-d+1)(j-d+2) .
$$

Note that we have $\left|G_{1}\right|+\left|G_{2}\right|+\ldots+\left|G_{n}\right|=\frac{(d-1)(d-2)}{2}$. Therefore, one can give an equivalent reformulation of the Conjecture 1 .

Conjecture 2. Suppose that the rational cuspidal curve $C$ of degree $d$ has critical points $z_{1}, \ldots, z_{n}$. Let $K_{1}, \ldots, K_{n}$ be the corresponding links of singular points and let $\Delta_{1}, \ldots, \Delta_{n}$ be their Alexander polynomials. Moreover, let $G_{1}, G_{2}, \ldots, G_{n}$ be the gap sequences of these points. Let $g=\left|G_{1}\right|+\left|G_{2}\right|+$ $\ldots+\left|G_{n}\right|$ be the genus of $K$. Let $\Delta=\Delta_{1}, \ldots, \Delta_{n}$, expanded as

$$
\Delta(t)=1+(t-1) g+(t-1)^{2} \sum_{j=0}^{2 g-2} k_{l}
$$

and let $I=I_{1} \diamond I_{2} \diamond \ldots \diamond I_{n}$. Then for any $j=0, \ldots, d-3$ we have $k_{d(d-j-3)} \leq$ $I(d(d-j-3)+1)$, with equality for $n=1$.

In this note we give an elementary argument, showing that FLMN conjecture follows from $[\mathrm{BL}]$ for $n=2$. The idea of our proof is to forget about the specific structure of the problem coming from theory of singularities and to prove Conjecture 2 for general sets $G_{1}, G_{2}$. Namely, we have the following theorem. 
Theorem 2. Let $G, H$ be two finite sets of positive integers and let $I_{G}, I_{H}$ : $\mathbb{Z} \rightarrow \mathbb{Z}_{\geq 0}$ be their gap functions. Let us define the polynomials

$$
\begin{aligned}
& \Delta_{G}(t)=1+(t-1) \sum_{j=1}^{|G|} t^{g_{j}}=1+(t-1)|G|+(t-1)^{2} \sum_{j \geq 0} k_{j}^{G} t^{j} \\
& \Delta_{H}(t)=1+(t-1) \sum_{j=1}^{|H|} t^{h_{j}}=1+(t-1)|H|+(t-1)^{2} \sum_{j \geq 0} k_{j}^{H} t^{j},
\end{aligned}
$$

where $k_{j}^{G}=I_{G}(j+1), k_{j}^{H}=I_{H}(j+1), j \geq 0$. Take $\Delta=\Delta_{G} \cdot \Delta_{H}$ and $I=I_{G} \diamond I_{H}$. Then

$$
\Delta(t)=1+(t-1)(|G|+|H|)+(t-1)^{2} \sum_{j \geq 0} k_{j} t^{j},
$$

where $k_{j} \leq I(j+1)$ for $j \geq 0$.

This gives the proof of Conjecture 1 in the case $n=2$.

It is natural to ask whether the above theorem is valid for arbitrary $n \geq 2$. Recently, after we found our elementary combinatorial argument for $n=2$, J. Bodnár and A. Némethi showed that the Conjecture 1 is false for $n \geq 3$, see $[\mathrm{BN}]$. They also found yet another proof of Conjecture 1 in the case of two singularities.

\section{Proof of the main result}

In this section we give a proof of our main result.

Proof. Our goal is to express the numbers $k_{j}$ in terms of the numbers $k_{j}^{G}$ and $k_{j}^{H}$. We have

$$
\begin{aligned}
& \Delta(t)=\Delta_{G}(t) \Delta_{H}(t)=1+(t-1)(|G|+|H|) \\
& +(t-1)^{2}\left[|G| \cdot|H|+\sum_{j \geq 0}\left(k_{j}^{G}+k_{j}^{H}\right) t^{j}+(t-1)\left(|G| \sum_{j \geq 0} k_{j}^{H} t^{j}+|H| \sum_{j \geq 0} k_{j}^{G} t^{j}\right)\right. \\
& \left.+(t-1)^{2}\left(\sum_{j \geq 0} k_{j}^{G} t^{j}\right)\left(\sum_{j \geq 0} k_{j}^{H} t^{j}\right)\right]=1+(t-1)(|G|+|H|)+(t-1)^{2} \Theta(t),
\end{aligned}
$$

with

$$
\Theta(t)=|G| \cdot|H|+k_{0}^{G}(1-|H|)+k_{0}^{H}(1-|G|)+k_{0}^{G} k_{0}^{H}+\sum_{j \geq 1} t^{j} k_{j},
$$

where

$$
k_{j}=k_{j}^{G}(1-|H|)+|H| k_{j-1}^{G}+k_{j}^{H}(1-|G|)+|G| k_{j-1}^{H}+l_{j}
$$


and

$$
l_{j}=\sum_{u+v=j, u, v \geq 0} k_{u}^{G} k_{v}^{H}-2 \sum_{u+v=j-1, u, v \geq 0} k_{u}^{G} k_{v}^{H}+\sum_{u+v=j-2, u, v \geq 0} k_{u}^{G} k_{v}^{H} .
$$

Note that $k_{0}^{G}=|G|$ and $k_{0}^{H}=|H|$. Therefore,

$$
\begin{aligned}
k_{0} & =|G| \cdot|H|+k_{0}^{G}(1-|H|)+k_{0}^{H}(1-|G|)+k_{0}^{G} k_{0}^{H} \\
& =|G| \cdot|H|+|G|(1-|H|)+|H|(1-|G|)+|G| \cdot|H|=|G|+|H| .
\end{aligned}
$$

Moreover, for $k \geq 1$ we have

$$
I_{H}(k) \geq|H|-(k-1), \quad I_{G}(1-k)=|G|+(k-1) .
$$

Thus, for $k \geq 1$ we obtain

$$
I_{G}(1-k)+I_{H}(k) \geq|G|+|H| .
$$

For $k \leq-1$

$$
I_{G}(1-k) \geq|G|+k, \quad I_{H}(k)=|H|-k .
$$

In this case we obtain

$$
I_{G}(1-k)+I_{H}(k) \geq|G|+|H|
$$

and we arrive at

$$
\begin{aligned}
I(1) & =\min _{k \in \mathbb{Z}}\left(I_{G}(1-k)+I_{H}(k)\right) \\
& =I_{G}(1)+I_{H}(0)=I_{G}(0)+I_{H}(1)=|G|+|H|=k_{0} .
\end{aligned}
$$

Note that

$$
\begin{aligned}
l_{j}= & \sum_{u+v=j, u, v \geq 0} k_{u}^{G} k_{v}^{H}-\sum_{u+v=j, u \geq 0, v \geq 1} k_{u}^{G} k_{v-1}^{H}-\sum_{u+v=j, u \geq 1, v \geq 0} k_{u-1}^{G} k_{v}^{H} \\
& +\sum_{u+v=j, u, v \geq 1} k_{u-1}^{G} k_{v-1}^{H}=\sum_{u+v=j, u, v \geq 1}\left(k_{u}^{G}-k_{u-1}^{G}\right)\left(k_{v}^{H}-k_{v-1}^{H}\right) \\
& +k_{0}^{G} k_{j}^{H}+k_{j}^{G} k_{0}^{H}-k_{0}^{G} k_{j-1}^{H}-k_{j-1}^{G} k_{0}^{H} .
\end{aligned}
$$

Thus,

$$
\begin{aligned}
k_{j}= & \sum_{u+v=j, u, v \geq 1}\left(k_{u}^{G}-k_{u-1}^{G}\right)\left(k_{v}^{H}-k_{v-1}^{H}\right) \\
& +\left(\left(k_{0}^{G} k_{j}^{H}+k_{j}^{G} k_{0}^{H}-k_{0}^{G} k_{j-1}^{H}-k_{j-1}^{G} k_{0}^{H}\right)\right. \\
& \left.+\left(k_{j}^{G}(1-|H|)+|H| k_{j-1}^{G}+k_{j}^{H}(1-|G|)+|G| k_{j-1}^{H}\right)\right) .
\end{aligned}
$$


Observe that we have a miracle,

$$
\begin{aligned}
k_{0}^{G} k_{j}^{H}+ & k_{j}^{G} k_{0}^{H}-k_{0}^{G} k_{j-1}^{H}-k_{j-1}^{G} k_{0}^{H} \\
\quad & \quad+k_{j}^{G}(1-|H|)+|H| k_{j-1}^{G}+k_{j}^{H}(1-|G|)+|G| k_{j-1}^{H} \\
=|G| k_{j}^{H}+ & k_{j}^{G}|H|-|G| k_{j-1}^{H}-k_{j-1}^{G}|H| \\
\quad & \quad k_{j}^{G}(1-|H|)+|H| k_{j-1}^{G}+k_{j}^{H}(1-|G|)+|G| k_{j-1}^{H} \\
= & k_{j}^{G}+k_{j}^{H} .
\end{aligned}
$$

We get

$$
k_{j}=k_{j}^{G}+k_{j}^{H}+\sum_{u+v=j, u, v \geq 1}\left(k_{u-1}^{G}-k_{u}^{G}\right)\left(k_{v-1}^{H}-k_{v}^{H}\right) .
$$

We are to prove that $k_{j} \leq\left(I_{G} \diamond I_{H}\right)(j+1)$. It suffices to prove that $k_{j} \leq I_{G}(j+1-l)+I_{H}(l)$ for every $l \in \mathbb{Z}$. Thus, we have to deal with the inequality

$k_{j}^{G}+k_{j}^{H}+\sum_{u+v=j, u, v \geq 1}\left(k_{u}^{G}-k_{u-1}^{G}\right)\left(k_{v}^{H}-k_{v-1}^{H}\right) \leq I_{G}(j+1-l)+I_{H}(l), \quad j \geq 1, l \in \mathbb{Z}$.

Note that if $u+v=j$ then we have either $u \geq j-l+1$ or $v \geq l$. Thus,

$$
\mathbf{1}_{u \in G} \mathbf{1}_{v \in H} \mathbf{1}_{u+v=j} \leq \mathbf{1}_{u \in G \cap[j-l+1, j]}+\mathbf{1}_{v \in H \cap[l, j]} .
$$

In the above expression we have used the convention $[a, b]=\emptyset$ for $a>b$. We obtain

$$
\begin{aligned}
\sum_{u+v=j, u, v \geq 1} & \left(k_{u-1}^{G}-k_{u}^{G}\right)\left(k_{v-1}^{H}-k_{v}^{H}\right)=\sum_{u+v=j, u, v \geq 0}\left(k_{u-1}^{G}-k_{u}^{G}\right)\left(k_{v-1}^{H}-k_{v}^{H}\right) \\
& =\sum_{u+v=j, u, v \geq 0} \mathbf{1}_{u \in G} \mathbf{1}_{v \in H} \leq \sum_{u+v=j, u, v \geq 0}\left(\mathbf{1}_{u \in G \cap[j-l+1, j]}+\mathbf{1}_{v \in H \cap[l, j]}\right) \\
& =\left(k_{j-l}^{G}-k_{j}^{G}\right)+\left(k_{l-1}^{H}-k_{j}^{H}\right)
\end{aligned}
$$

what finishes our proof.

\section{Acknowledgements}

We are grateful to Maciej Borodzik for his valuable comments about the meaning of our result. 


\section{References}

[FLMN] J. Fernández de Bobadilla, I. Luengo, A. Melle-Hernández, A. Némethi, Classification of rational unicuspidal projective curves whose singularities have one Puiseux pair, Proceedings of Sao Carlos Workshop 2004 Real and Complex Singularities, Series Trends in Mathematics, Birkhäuser 2007, 31-46.

[BL] M. Borodzik, C. Livingston, Heegaard Floer homology and rational cuspidal curves, arXiv:1304.1062

[BN] J. Bodnár and A. Némethi, arXiv:1405.0437

[M] K. Moe, Rational cuspidal curves, Master Thesis, University of Oslo 2008, permanent link at University of Oslo:

https://www.duo.uio.no/handle/123456789/10759

[W] C. T. C. Wall, Singular Points of Plane Curves, London Mathematical Society Student Texts, 63. Cambridge University Press, Cambridge, 2004.

Piotr Nayar, nayar@mimuw.edu.pl

Institute of Mathematics, University of Warsaw,

Banacha 2,

02-097 Warszawa,

Poland.

Barbara Pilat, B.Pilat@mini.pw.edu.pl

Faculty of Mathematics and Information Science, Warsaw University of Technology,

Koszykowa 75 ,

00-662 Warszawa,

Poland. 